\title{
Efeitos das condições ambientais no período entre a postura e o armazenamento de ovos de matrizes pesadas sobre o rendimento de incubação
}

[Effect on time between oviposition and the beginning of cool storage on hatchability of broiler breeder eggs]

\author{
M.A. Fiuza, L.J.C. Lara, C.A.L. Aguilar, B.R.C. Ribeiro, N.C. Baião* \\ Escola de Veterinária - UFMG \\ Caixa postal 567 \\ 30123-970 - Belo Horizonte, MG
}

\begin{abstract}
RESUMO
Avaliou-se o efeito das condições ambientais no período entre a postura e o armazenamento de ovos sobre o rendimento de incubação de 2.742 ovos de matrizes pesadas da linhagem Ross com 31 semanas de idade. Os tratamentos foram definidos pelo período de permanência dos ovos sob temperatura ambiente do galpão antes do armazenamento. Para cada tratamento foram utilizados 860 ovos, distribuídos em 10 repetições de 86 ovos cada. No tratamento A, os ovos foram enviados para a sala fria imediatamente após a coleta; no B, foram enviados para a sala fria após cinco horas de permanência no galpão; e, no C, permaneceram no galpão por 10 horas, antes de serem enviados para a sala fria. As condições ambientais da sala fria foram: temperatura 18,2 a $21,0{ }^{\circ} \mathrm{C}$ e umidade relativa do ar de 72,8 a $76,8 \%$. O período de armazenamento dos ovos foi de quatro dias. A mortalidade embrionária no tratamento $\mathrm{A}$ foi maior que no tratamento $\mathrm{B}(\mathrm{P}<0,05)$. A eclosão dos ovos do tratamento $\mathrm{B}$ foi maior que aquela do tratamento $\mathrm{A}(\mathrm{P}<0,05)$. $\mathrm{O}$ peso dos pintos do tratamento $\mathrm{A}$ foi maior que aqueles do tratamento $\mathrm{C}(\mathrm{P}<0,05)$. $\mathrm{O}$ melhor rendimento de incubação foi obtido quando os ovos foram resfriados cinco horas após a postura.
\end{abstract}

Palavras-chave: matriz pesada, ovo, armazenamento, eclosão, mortalidade embrionária

\section{ABSTRACT}

The effect on hatchability of time post-oviposition until initiation of cool storage was examined using 2,742 eggs from 31-week-old Ross hens. Treatments were defined by the length of time that eggs remained at breeder house temperature, prior to cool storage. In treatments $A, B$ and $C$, respectively, eggs were taken to the cooling room immediately after collection, after 5 hours in the aviary or after 10 hours in the aviary. From each treatment, 860 eggs were distributed into 10 replicates of 86 eggs each and stored for 4 days at $18.2-21.0^{\circ} \mathrm{C}$ and $75 \%$ humidity. After cool storage, they were incubated. Embryo mortality in treatment $A$ was higher than in $B(P<0.05)$. The hatchability of eggs of treatment $B$ was higher $(P<0.05)$ than that of treatment A. Average chick weight at hatching was higher for treatment $A$ than for treatment $C(P<0.05)$. Eggs that were cooled beginning five hours after oviposition produced the highest incubation yield.

Keywords: broiler breeder, egg, hatchability, embryo mortality, cool storage

Recebido em 29 de julho de 2004

Aceito em 28 de março de 2005

*Autor para correspondência (corresponding author)

E-mail: baiao@vet.ufmg.br 


\section{INTRODUÇÃO}

A fertilização do óvulo de galinha ocorre no infundíbulo, logo após a ovulação, e o processo de formação do ovo a partir da ovulação demora cerca de 24 horas. Durante esse período, o ovo permanece no oviduto, onde a temperatura é de aproximadamente $42^{\circ} \mathrm{C}$, possibilitando $\mathrm{o}$ desenvolvimento embrionário, que, no momento da postura, encontra-se no estádio de prégástrula. $\mathrm{O}$ ovo fertilizado mantido em temperaturas elevadas favorece o contínuo desenvolvimento do embrião. Este desenvolvimento é paralisado quando o ovo é colocado à temperatura ambiente abaixo do ponto zero fisiológico que, segundo Fasenko et al. (1991), está em torno de $20-21^{\circ} \mathrm{C}$.

Em condições de campo, freqüentemente, observa-se queda na eclosão dos ovos de matriz pesada nas épocas quentes do ano. A análise dos ovos não eclodidos normalmente revela alta taxa de mortalidade embrionária nos primeiros dias de incubação. Segundo Johar et al. (1973), a exposição do ovo à temperatura elevada antes do armazenamento é prejudicial ao desenvolvimento embrionário.

De acordo com North e Bell (1990), em épocas de temperatura ambiente elevada, a colheita de ovos deve ser feita cinco a seis vezes por dia, e a armazenagem deve ser sob temperatura de $18^{\circ} \mathrm{C}$ por até cinco dias. Quando esse período for maior que cinco dias, a temperatura deve ser de $12-13^{\circ} \mathrm{C}$ (Fasenko et al., 1992).

Os embriões que, logo após a ovoposição, encontram-se no estágio de pré-gástrula são menos resistentes ao estresse de armazenamento do que embriões no estágio de gástrula. $\mathrm{O}$ efeito dessa desvantagem pode ser diminuído mediante aquecimento dos ovos logo após a postura (Butler, 1991).

O objetivo deste trabalho foi verificar o efeito das condições ambientais, no período entre a postura e o armazenamento dos ovos sobre: a temperatura interna do ovo durante o resfriamento, o peso do ovo pós-armazenamento, a perda de peso do ovo durante a incubação, a mortalidade embrionária, a eclosão, o peso do pinto e a relação peso do pinto ao nascer e peso do ovo.

\section{MATERIAL E MÉTODOS}

Foram utilizados 2.742 ovos incubáveis provenientes de um lote de matrizes pesadas da linhagem Ross - 308, com 31 semanas de idade. Os ovos foram obtidos da segunda coleta do dia, realizada às $9 \mathrm{~h} 30$.

Os tratamentos foram definidos com base no tempo de esfriamento dos ovos entre a postura e o armazenamento, caracterizado pelo tempo de permanência dos ovos em temperatura ambiente do galpão aberto (convencional) onde os ovos foram produzidos. Os tratamentos foram: tratamento A - após a coleta, os ovos foram imediatamente enviados para a sala fria, onde a temperatura ambiente $\mathrm{e}$ a umidade do ar oscilaram entre 18,2 e $21,0^{\circ} \mathrm{C}$ e 72,8 e $76,8 \%$ respectivamente; tratamento $\mathrm{B}$ - após a coleta, os ovos permaneceram no galpão durante cinco horas, onde a temperatura ambiente e a umidade relativa do ar oscilaram entre 29,3 e $31,7^{\circ} \mathrm{C}$ e 55,0 e $60,1 \%$ respectivamente, e depois foram enviados para a mesma sala fria do tratamento $\mathrm{A}$; tratamento C - após a coleta, os ovos permaneceram no galpão durante 10 horas, onde a temperatura ambiente e a umidade relativa do ar variaram entre 26,0 e $31,7^{\circ} \mathrm{C}$ e 60,1 e $77,0 \%$ respectivamente, e depois foram enviados para a mesma sala fria do tratamento A.

Imediatamente após a coleta, uma amostra de 18 ovos foi aleatoriamente colhida para determinar a temperatura interna deles feita com termômetro digital com sonda de penetração. Este momento foi considerado o início do controle da temperatura interna dos ovos correspondentes ao tratamento A. Para verificar a equiparação da temperatura interna do ovo com a do ambiente, após sua colocação na sala fria, a cada cinco horas foi tomada a temperatura interna de uma amostra aleatória de 18 ovos, de acordo com os tratamentos. Os ovos do tratamento A foram amostrados após cinco, 10 e 15 horas de permanência na sala fria; os do tratamento B, com cinco e 10 horas de armazenamento, e a única amostragem do tratamento $\mathrm{C}$ foi realizada com cinco horas de permanência na sala fria.

Antes de serem incubados, os ovos passaram por um pré-aquecimento de seis horas na sala de incubação. 
Mediram-se: temperatura interna dos ovos; redução total da temperatura interna $\left({ }^{\circ} \mathrm{C}\right)$ e redução média de sua temperatura interna, durante as primeiras cinco horas de resfriamento; peso médio dos ovos pós-armazenamento; percentual de perda de peso médio dos ovos (inicial- zero a oito dias de incubação, final - oito a 18 dias de incubação e total - zero a 18 dias de incubação); peso médio dos pintos ao nascimento; relação peso médio dos pintos ao nascimento e peso médio dos ovos pósarmazenamento; percentual de fertilidade; percentual de mortalidade embrionária de acordo com as fases (inicial - zero a 11 dias, final - 12 a 21 dias, bicados vivos e mortos); correlação entre o peso médio do pinto ao nascimento e o peso médio do ovo pós-armazenamento; percentual de eclosão total; percentual de eclosão dos ovos férteis.

Para as avaliações referentes à incubação, o delineamento experimental foi inteiramente ao acaso, com três tratamentos e 10 repetições, sendo cada repetição constituída por uma bandeja com 86 ovos. Para avaliar a temperatura do ovo no galpão e na sala fria, o delineamento experimental foi o mesmo, mas neste caso foi utilizada uma amostra de 18 ovos (cada ovo foi considerado como uma repetição) para cada avaliação, totalizando 72,54 e 36 ovos para os tratamentos A, B e C, respectivamente (Sampaio, 2002). Realizou-se a análise de variância para cada característica estudada, e as diferenças entre os tratamentos foram avaliadas através do teste Duncan, utilizando o programa SAS (User`s... 1990).

\section{RESULTADOS E DISCUSSÃO}

Os dados da temperatura interna do ovo da coleta até sua equiparação com a temperatura ambiente da sala fria são apresentados na Tab. 1. Nela são apresentados, também, os dados referentes às condições ambientais da sala fria e do galpão de produção, nos momentos em que foram tomadas as medidas de temperatura dos ovos, de acordo com os tratamentos.

Tabela 1. Temperatura $\left({ }^{\circ} \mathrm{C}\right)$ interna dos ovos de acordo com os tratamentos e dados das condições ambientais da sala fria do galpão

\begin{tabular}{lcccc}
\hline \multirow{2}{*}{ Tratamento } & \multicolumn{4}{c}{ Horário da amostragem } \\
\cline { 2 - 5 } & 10 & 15 & 20 & 1 \\
\hline TA & $34,25 \mathrm{a}$ & $21,70 \mathrm{~b} \mathrm{~B}$ & $21,60 \mathrm{~b} \mathrm{~B}$ & $21,60 \mathrm{~b} \mathrm{~A}$ \\
TB & - & $32,30 \mathrm{a} \mathrm{A}$ & $21,70 \mathrm{~b} \mathrm{~B}$ & $21,70 \mathrm{~b} \mathrm{~A}$ \\
TC & - & - & $29,30 \mathrm{a} \mathrm{A}$ & $21,60 \mathrm{a} \mathrm{A}$ \\
Temperatura da sala fria $\left({ }^{\circ} \mathrm{C}\right)$ & 20,00 & 21,00 & 20,00 & 18,20 \\
Umidade do ar da sala fria $(\%)$ & 74,80 & 72,80 & 75,20 & 76,70 \\
Temperatura do galpão $\left({ }^{\circ} \mathrm{C}\right)$ & 29,30 & 31,70 & 26,00 & - \\
Umidade do ar do galpão $(\%)$ & 60,10 & 55,00 & 77,00 & - \\
\hline
\end{tabular}

Letras distintas, minúsculas na linha e maiúsculas na coluna, indicam diferenças entre os valores $(\mathrm{P}<0,05)$.

$\mathrm{TA}=$ ovos enviados imediatamente para sala fria; $\mathrm{TB}=$ ovos enviados para sala fria após $5 \mathrm{~h}$; $\mathrm{TC}=$ ovos enviados para sala fria após 10h. * Coeficiente de variação $=2,2 \%$.

$\mathrm{Na}$ amostragem das $15 \mathrm{~h}$, observou-se que a temperatura interna dos ovos do tratamento B foi maior que a registrada nos ovos do tratamento $\mathrm{A}$ $(\mathrm{P}<0,05)$. Na amostragem das $20 \mathrm{~h}, \mathrm{o}$ TC foi diferente do TA e TB. Na amostragem de $1 \mathrm{~h}$, todos os ovos encontravam-se dentro da sala fria, e as temperaturas internas deles foram semelhantes entre si $(\mathrm{P}>0,05)$. Para todos os tratamentos, a temperatura interna dos ovos foi estabilizada após cinco horas de permanência na sala fria. Considerando que os valores de estabilização da temperatura interna dos ovos foram muito próximos aos obtidos na sala fria, pode-se sugerir que o tempo gasto para que haja a equiparação da temperatura interna do ovo com a temperatura do ambiente seja de cinco horas.

Os valores referentes à redução total da temperatura interna dos ovos e redução média por hora, apresentados na Tab. 2, mostram que, durante o período de estabilização da temperatura interna do ovo, a redução de temperatura interna no tratamento $\mathrm{A}$ foi maior que as verificadas nos ovos dos tratamentos $\mathrm{B}$ e C $(\mathrm{P}<0,05)$; também foram significativas as diferenças entre as temperaturas dos ovos dos tratamentos B e C. 
Tabela 2. Redução total da temperatura interna do ovo $\left({ }^{\circ} \mathrm{C}\right)$ e redução / hora durante as primeiras cinco horas na sala fria

\begin{tabular}{lccc}
\hline \multirow{2}{*}{ Redução } & \multicolumn{3}{c}{ Tratamento } \\
\cline { 2 - 4 } & $\mathrm{A}$ & $\mathrm{B}$ & $\mathrm{C}$ \\
\hline Total & $12,55 \mathrm{a}$ & $10,60 \mathrm{~b}$ & $7,7 \mathrm{c}$ \\
Média/hora & $2,5 \mathrm{a}$ & $2,1 \mathrm{~b}$ & $1,5 \mathrm{c}$ \\
\hline
\end{tabular}

Letras distintas na linha indicam diferenças entre os valores $(\mathrm{P}<0,05)$ pelo teste de Duncan.

$\mathrm{TA}=$ ovos enviados imediatamente para sala fria $\mathrm{TB}=$ ovos enviados para sala fria após $5 \mathrm{~h}$; $\mathrm{TC}=$ ovos enviados para sala fria após $10 \mathrm{~h}$. Coeficiente de variação $=7,3 \%$.

As diferenças na redução da temperatura interna dos ovos, durante a estabilização, podem ser explicadas pela observação dos dados apresentados na Tab. 1. No início do resfriamento (10 horas), a temperatura interna dos ovos do tratamento A foi maior que a do tratamento B, e a desse maior que a dos ovos do tratamento C. Após a estabilização, todas as temperaturas tornaram-se semelhantes. Essa equiparação ocorreu porque os ovos, cuja temperatura foi mais elevada no início do armazenamento, transferiram maior quantidade de calor para o ambiente nesse período.

Nos oito primeiros dias de incubação, a perda de peso dos ovos do tratamento $\mathrm{A}$ foi maior $(\mathrm{P}<0,05)$ do que a dos ovos dos tratamentos $\mathrm{B}$ e $\mathrm{C}$, os quais foram semelhantes entre si (Tab. 3). Este mesmo comportamento foi observado para as perdas de peso de zero a 18 dias de incubação e para a perda de peso média diária. Entretanto, no período de oito a 18 dias de incubação, não foram observadas diferenças significativas entre os tratamentos.

Tabela 3. Percentual de perda de peso médio dos ovos do início da incubação até a transferência para o nascedouro de acordo com os tratamentos

\begin{tabular}{lcccc}
\hline \multirow{2}{*}{ Período de incubação (dias) } & \multicolumn{3}{c}{ Tratamento } \\
\cline { 2 - 5 } & $\mathrm{A}$ & $\mathrm{B}$ & $\mathrm{C}$ & $\mathrm{CV}(\%)$ \\
\hline Inicial (0 a 8) & $4,87 \mathrm{a}$ & $4,62 \mathrm{~b}$ & $4,64 \mathrm{~b}$ & 4,00 \\
Final (8 a 18) & $6,81 \mathrm{a}$ & $6,63 \mathrm{a}$ & $6,66 \mathrm{a}$ & 3,47 \\
Total (0 a 18) & $11,68 \mathrm{a}$ & $11,25 \mathrm{~b}$ & $11,30 \mathrm{~b}$ & 3,14 \\
Perda média de peso / dia & $0,65 \mathrm{a}$ & $0,62 \mathrm{~b}$ & $0,63 \mathrm{~b}$ & 3,14 \\
\hline
\end{tabular}

Letras distintas na linha indicam diferenças entre os valores $(\mathrm{P}<0,05)$ pelo teste Duncan.

$\mathrm{TA}=$ ovos enviados imediatamente para sala fria; $\mathrm{TB}=$ ovos enviados para sala fria após $5 \mathrm{~h}$; $\mathrm{TC}=$ ovos enviados para sala fria após 10h. $\mathrm{CV}=$ coeficiente de variação.

O resultado da maior perda diária de peso dos ovos do tratamento A pode estar relacionado com o tempo de permanência deles no galpão. Como eles permaneceram menos tempo no galpão (onde a temperatura foi maior do que a da sala fria) do que os ovos dos tratamentos B e C, é provável que tenha havido menor desidratação nessa etapa, compensada pela maior desidratação nos primeiros oito dias de incubação. Os percentuais de perda de peso médio de 11,3 a
$11,7 \%$ obtidos nos primeiros 18 dias de incubação estão dentro dos limites preconizados por Mauldin (1993), assim como o percentual médio de perda diária de peso.

Mesmo sabendo que o manejo dos ovos não afeta a fertilidade, essa variável foi analisada com o objetivo de demonstrar que os ovos submetidos aos diferentes tratamentos tinham índices semelhantes de fertilidade (Tab. 4).

Tabela 4. Percentual de fertilidade, mortalidade embrionária, eclodibilidade total dos ovos incubados e eclodibilidade dos ovos férteis de acordo com os tratamentos

\begin{tabular}{lcccc}
\hline \multirow{2}{*}{ Variáveis } & \multicolumn{3}{c}{ Tratamento } \\
\cline { 2 - 5 } & $\mathrm{A}$ & $\mathrm{B}$ & $\mathrm{C}$ & $\mathrm{CV} *$ \\
\hline Fertilidade & $97,91 \mathrm{a}$ & $97,21 \mathrm{a}$ & $96,86 \mathrm{a}$ & 1,75 \\
Mort. embrionária inicial & $6,98 \mathrm{~b}$ & $4,07 \mathrm{a}$ & $5,35 \mathrm{a} \mathrm{b}$ & 34,96 \\
Mort. embrionária final & $3,14 \mathrm{a}$ & $2,91 \mathrm{a}$ & $2,44 \mathrm{a}$ & 69,62 \\
Bicados vivos e mortos & $4,13 \mathrm{a}$ & $3,39 \mathrm{a}$ & $3,84 \mathrm{a}$ & 66,24 \\
Mort. embrionária total & $14,25 \mathrm{~b}$ & $10,37 \mathrm{a}$ & $11,63 \mathrm{a} \mathrm{b}$ & 27,47 \\
Eclodibilidade total & $83,65 \mathrm{~b}$ & $86,84 \mathrm{a}$ & $85,22 \mathrm{a} \mathrm{b}$ & 3,85 \\
Eclodibilidade dos férteis & $85,75 \mathrm{~b}$ & $89,63 \mathrm{a}$ & $88,36 \mathrm{a} \mathrm{b}$ & 3,99 \\
\hline
\end{tabular}

Letras distintas na linha indicam diferenças entre os valores $(\mathrm{P}<0,05)$ pelo teste Duncan.

$\mathrm{TA}=$ ovos enviados imediatamente para sala fria; $\mathrm{TB}=$ ovos enviados para sala fria após $5 \mathrm{~h}$; TC $=$ ovos enviados para sala fria após $10 \mathrm{~h}$. CV= coeficiente de variação. 
A maior mortalidade embrionária inicial ocorreu nos ovos do tratamento $\mathrm{A}$, superior àquela observada no tratamento B $(\mathrm{P}<0,05)$, mas semelhante à encontrada nos ovos do tratamento $\mathrm{C}$; os tratamentos $\mathrm{B}$ e $\mathrm{C}$ não diferiram entre si $(\mathrm{P}>0,05)$. $\mathrm{O}$ efeito dos tratamentos sobre a mortalidade embrionária total teve o mesmo comportamento do verificado para a mortalidade embrionária inicial. De acordo com Butler (1991), embriões no estágio de pré-gástrula, próximo à ovoposição, são mais sensíveis ao estresse de armazenamento do que aqueles com estágio de gastrulação mais avançado. Essa afirmativa pode ser aplicada neste experimento, em que os ovos do tratamento A, provavelmente, tinham embriões menores e mais sensíveis ao estresse de resfriamento do que aqueles dos tratamentos $\mathrm{B}$ e $\mathrm{C}$, porque $\mathrm{O}$ período de exposição dos ovos do tratamento $\mathrm{A}$ à temperatura ambiente acima do ponto de zero fisiológico foi, consideravelmente, menor que o dos ovos dos tratamentos B e C. Desse modo, a redução de temperatura interna foi mais rápida nos ovos do tratamento $\mathrm{A}$ em relação ao tratamento B. Pode-se deduzir que, possivelmente, essa associação foi responsável pelo maior percentual de mortalidade embrionária inicial observado no tratamento $\mathrm{A}$ em relação ao tratamento $\mathrm{B}$ durante a incubação. Estes resultados foram diferentes dos obtidos por Bowling e Howarth (1981), que, antes do armazenamento por quatro dias a $15^{\circ} \mathrm{C}$, mantiveram os ovos sob temperatura de 24 ou $35^{\circ} \mathrm{C}$ por 3,6 e 12 horas e concluíram que os tratamentos não afetaram a mortalidade embrionária nos primeiros 11 dias de incubação.
Os percentuais de mortalidade embrionária final (12 a 21 dias de incubação) dos tratamentos A, B e $\mathrm{C}$ foram semelhantes entre si $(\mathrm{P}>0,05)$ (Tab. 4). Este resultado foi semelhante ao obtido por Bowling e Howarth (1981), que não verificaram efeito dos tratamentos sobre a mortalidade embrionária nos últimos 10 dias de incubação.

As taxas de ovos bicados com embriões vivos e mortos dos tratamentos A, B e C foram semelhantes entre si $(\mathrm{P}>0,05)$.

A eclodibilidade em relação ao total de ovos incubados e em relação aos ovos férteis do tratamento $\mathrm{B}$ foi maior que a do tratamento $\mathrm{A}$ $(\mathrm{P}<0,05)$, sendo a eclodibilidade do tratamento $\mathrm{C}$ intermediária e semelhante a A e B. A maior taxa de eclodibilidade dos ovos do tratamento $\mathrm{B}$ em relação à do tratamento $\mathrm{A}$ está relacionada, principalmente, à menor mortalidade embrionária inicial $(\mathrm{P}<0,05)$, obtida no tratamento $\mathrm{B}$ em comparação à obtida no tratamento A. Os resultados deste trabalho divergem dos obtidos por Heymang (1945), que verificou eclodibilidade semelhante entre os ovos férteis resfriados imediatamente após coleta e aqueles mantidos sob temperatura ambiente entre 32 e $40^{\circ} \mathrm{C}$ até às $17 \mathrm{~h}$ do dia de coleta no período pré-armazenamento. Essa divergência pode estar relacionada à grande diferença entre as aves utilizadas.

O peso médio dos ovos pós-armazenamento (Tab. 5) foi semelhante entre os tratamentos $(\mathrm{P}>0,05)$, evidenciando que os tratamentos não tiveram influência sobre essa variável.

Tabela 5. Peso médio do ovo pós-armazenamento, peso médio do pinto ao nascimento e a relação percentual peso médio do pinto / peso médio do ovo de acordo com os tratamentos

\begin{tabular}{|c|c|c|c|c|}
\hline \multirow{2}{*}{ Variável } & \multicolumn{4}{|c|}{ Tratamento } \\
\hline & A & $\mathrm{B}$ & $\mathrm{C}$ & $\mathrm{CV}(\%)$ \\
\hline Peso médio do ovo $(\mathrm{g})$ & $59,20 \mathrm{a}$ & $59,00 \mathrm{a}$ & $58,80 \mathrm{a}$ & 1,03 \\
\hline Peso médio do pinto $(\mathrm{g})$ & $42,20 \mathrm{a}$ & $41,90 \mathrm{ab}$ & $41,54 b$ & 1,41 \\
\hline Relação peso médio do pinto/ peso médio do ovo & $71,28 \mathrm{a}$ & $71,02 \mathrm{a}$ & $70,65 \mathrm{a}$ & 1,01 \\
\hline
\end{tabular}

Letras distintas na linha indicam diferenças entre valores $(\mathrm{P}<0,05)$ pelo teste Duncan.

$\mathrm{TA}=$ ovos enviados imediatamente para sala fria; $\mathrm{TB}=$ ovos enviados para sala fria após $5 \mathrm{~h} ; \mathrm{TC}=$ ovos enviados para sala fria após 10h. $\mathrm{CV}=$ coeficiente de variação.

Os pintos originados dos ovos do tratamento A foram significativamente mais pesados que os do tratamento $\mathrm{C}(\mathrm{P}>0,05)$, e o peso médio dos pintos do tratamento $\mathrm{B}$ foi intermediário e semelhante aos dos tratamentos A e C. Estes resultados demonstraram que o peso médio do pinto ao nascimento foi $(\mathrm{P}<0,05)$ influenciado pelo período de permanência dos ovos no galpão 
antes do armazenamento, entretanto não foi possível evidenciar como os tratamentos influenciaram o peso médio do pinto no nascimento.

Os tratamentos não afetaram $(\mathrm{P}>0,05)$ os percentuais da relação entre o peso médio do pinto ao nascer e o peso médio do ovo pósarmazenamento. Estes resultados foram semelhantes aos obtidos por Barbosa (1992), que não observou efeito das condições ambientais de armazenamento dos ovos sobre essa característica, e superiores ao valor médio de $68,0 \%$ indicado por Shanawany (1987).

Observou-se correlação positiva significativa entre o peso médio do pinto ao nascimento e o peso médio do ovo pós-armazenamento, sendo de 0,$64 ; 0,84$ e 0,77 para os tratamentos A, B e $\mathrm{C}$, respectivamente. Estes resultados foram semelhantes aos descritos por Washburn e Guill (1974) e Wilson (1991).

\section{CONCLUSÕES}

Conclui-se que a estabilização da temperatura interna dos ovos na sala fria ocorre nas primeiras cinco horas de resfriamento; o resfriamento dos ovos imediatamente após a coleta é prejudicial ao embrião e, conseqüentemente, à eclodibilidade dos ovos; a relação peso do pinto ao nascimento e peso do ovo pós-armazenamento não é influenciada pelas condições ambientais do período pré-armazenamento; o período de cinco horas de permanência dos ovos no galpão é indicado para melhorar o rendimento de incubação.

\section{REFERÊNCIAS BIBLIOGRÁFICAS}

BARBOSA, M.A.S. Efeitos da temperatura, umidade relativa do ar e do periodo de armazenamento de ovos de matrizes pesadas, sobre o rendimento de incubação. 1992. 39f. Dissertação (Mestrado em Zootecnia) - Escola de Veterinária, Universidade Federal de Minas Gerais, Belo Horizonte.

BOWLING, J.A.; HOWARTH JR., B. The effects of exposing broiler breeder eggs to high temperature before storage on hatchability and subsequent performance of chicks. Poult. Sci., v.60, p. 2333 - 2336, 1981.

BUTLER, D.E. Egg handling and storage at the farm and hatchery. In: TULLET, S.G. (Ed.). Avian incubation. London: Butteworth Heinemanny, 1991. p.195-203.

FASENKO, G.M.; HARDIN, R.T.; ROBINSON, F.E. et al. Relationship of hen age and sequence position with fertility, hatchability, viability and preincubation embryonic development in broiler breeders. Poult. Sci., v.71, p.1374 - 1384, 1992.

FASENKO, G.M.; ROBINSON, F.E.; ARMSTRONG, J.G. et al. Variability in preincubation embryo development in domestic fowl: Effects of nest holding time and method of egg storage. Poult. Sci., v.70, p.1876 - 1881, 1991.

HEYMANG, B.W. Gathering and storing hatching eggs during hot weather. Poult. Sci., v. 24, p. $434-437,1945$.

JOHAR, K.S.; BRAY, B.D.J.; NORTON, H.W. Effects of holding temperature on hatchability of White Leghorn an New Hampshire eggs. Indian Vet. J., v.50, p.66 - 71, 1973.

MAULDIN, J.M. Hatchery management. Int. Hatch. Pract., v.8, p.47, 1993.

NORTH, M.O.; BELL, D.D. Maintaing hatching egg quality. In:_. Commercial chicken production manual. New York: Chapman \& Hall, 1990. p.31 - 44.

SAMPAIO, I.B.M. Estatística aplicada à experimentação animal. 2.ed. Belo Horizonte: FEPMVZ, 2002. 264p.

SHANAWANY, M.M. Hatching weight in relation to egg weight in domestic birds. World's Poult. Sci., v.43, p.107 - 115, 1987.

USER'S guide: statistics. Version. 6.0. 3.ed. Cary, NC: SAS Institute, 1990.

WASHBURN, K.W.; GUILL, R.A. Relationship of embryo weight as a percent of egg weight to efficiency of feed utilization in the hatched chick. Poult. Sci., v.53, p.766-769, 1974.

WILSON, H.R. Interrelationships of egg size, chick size, posthatching and hatchability. World's Poult. Sci.,, v.47, p.5-20, 1991. 